\title{
Predicting the response to neoadjuvant chemotherapy for breast cancer: wavelet transforming radiomics in MRI
}

Jiali Zhou ${ }^{1,2,3 \dagger}$, Jinghui Lu ${ }^{4+}$, Chen Gao ${ }^{1,2}$, Jingjing Zeng ${ }^{5}$, Changyu Zhou ${ }^{1,2}$, Xiaobo Lai ${ }^{1,2}$, Wenli Cai ${ }^{4^{* \dagger}}$ and Maosheng $\mathrm{Xu}^{1,2^{*}+}(\mathrm{D}$

\begin{abstract}
Background: The purpose of this study was to investigate the value of wavelet-transformed radiomic MRI in predicting the pathological complete response ( $\mathrm{pCR}$ ) to neoadjuvant chemotherapy (NAC) for patients with locally advanced breast cancer (LABC).

Methods: Fifty-five female patients with $L A B C$ who underwent contrast-enhanced MRI (CE-MRI) examination prior to NAC were collected for the retrospective study. According to the pathological assessment after NAC, patient responses to NAC were categorized into $\mathrm{pCR}$ and non-pCR. Three groups of radiomic textures were calculated in the segmented lesions, including (1) volumetric textures, (2) peripheral textures, and (3) wavelet-transformed textures. Six models for the prediction of pCR were Model I: group (1), Model II: group (1) + (2), Model III: group (3), Model IV: group (1) + (3), Model V: group (2) + (3), and Model VI: group (1) + (2) + (3). The performance of predicting models was compared using the area under the receiver operating characteristic (ROC) curves (AUC).

Results: The AUCs of the six models for the prediction of pCR were $0.816 \pm 0.033$ (Model I), $0.823 \pm 0.020$ (Model II), $0.888 \pm 0.025$ (Model III), $0.876 \pm 0.015$ (Model IV), $0.885 \pm 0.030$ (Model V), and $0.874 \pm 0.019$ (Model VI). The performance of four models with wavelet-transformed textures (Models III, IV, V, and VI) was significantly better than those without wavelet-transformed textures (Model I and II). In addition, the inclusion of volumetric textures or peripheral textures or both did not result in any improvements in performance.

Conclusions: Wavelet-transformed textures outperformed volumetric and/or peripheral textures in the radiomic MRI prediction of $p C R$ to NAC for patients with $\angle A B C$, which can potentially serve as a surrogate biomarker for the prediction of the response of $L A B C$ to NAC.
\end{abstract}

Keywords: Radiomics, Breast cancer, Neoadjuvant chemotherapy, Pathological complete response

\section{Background}

Breast cancer is the most common malignant tumor among women across the world [1, 2]. For treatment, preoperative neoadjuvant chemotherapy (NAC) plays a major role in patients with locally advanced breast

\footnotetext{
* Correspondence: Cai.Wenli@mgh.harvard.edu; xums166@zcmu.edu.cn †Jiali Zhou and Jinghui Lu contributed equally to the first authors.

${ }^{\dagger}$ Maosheng Xu and Wenli Cai contributed equally to the corresponding authors. ${ }^{4}$ Department of Radiology, Massachusetts General Hospital and Harvard Medical School, 25 New Chardon St., 400C, Boston, MA 02114, USA

'Department of Radiology, The First Affiliated Hospital of Zhejiang Chinese Medical University, 54 Youdian Road, Shangcheng District, Hangzhou 310006, People's Republic of China

Full list of author information is available at the end of the article
}

cancer (LABC) [3]. With proper therapy, NAC has been shown to decrease tumor size, downstage tumors, and allow breast-conserving surgery to take place with clearer margins [4]. Furthermore, timely NAC therapy can also improve the efficacy of follow-up treatment options after surgery [5].

The response of breast cancer to NAC relies on the post-treatment pathology, and the pathological complete response $(\mathrm{pCR})$ is clinically defined as having no residual invasive carcinoma in the breast tissue after surgery, which is associated with a better prognosis [6]. However, it has been reported that the pCR rate of NAC for breast cancer varies between 10 and 50\% [7]. This poor pCR

(c) The Author(s). 2020 Open Access This article is distributed under the terms of the Creative Commons Attribution 4.0 International License (http://creativecommons.org/licenses/by/4.0/), which permits unrestricted use, distribution, and reproduction in any medium, provided you give appropriate credit to the original author(s) and the source, provide a link to the Creative Commons license, and indicate if changes were made. The Creative Commons Public Domain Dedication waiver (http://creativecommons.org/publicdomain/zero/1.0/) applies to the data made available in this article, unless otherwise stated. 
rate signifies that the majority of patients receiving NAC may benefit from a treatment course other than NAC. Given the well-documented adverse effects to chemotherapy [8], an urgent clinical need is present for objective surrogate biomarkers to accurately predict the response of breast cancer to NAC.

Radiomics is an emerging technology in quantitative imaging analysis, which hypothesizes that the spatial tumor heterogeneity is related to tissue changes on histological analysis. Preliminary studies using radiomics for breast MRI have shown that certain pre-treatment texture parameters (based on high order statistics) may help in evaluating breast tumor response to NAC [9-12]. Previously, high throughput image textures have been obtained for radiomics analysis to predict the efficacy of NAC prior to initiating treatment $[13,14]$. The majority of studies applied morphologic features and gray-level textures (such as histogram, gray-level co-occurrence matrices, etc) extracted from regions of interest (ROI). Alternatively, wavelet transformation can provide comprehensive spatial, and frequency distributions for characterizing intratumoral and peritumoral regions in terms of low and high frequency signals. These properties may improve the performance of radiomic model $[15,16]$. The aim of this study was to investigate whether wavelet-transformed textures can improve the performance of radiomic MRI predictions of pCR to NAC in comparison to those utilizing various combinations of volumetric textures, peripheral textures, and wavelet-transformed textures extracted in breast MRI.

\section{Methods \\ Patients}

The Ethics Committee of the First Affiliated Hospital of Zhejiang Chinese Medical University has approved this retrospective study, in which informed consent was waived, but patient confidentiality was protected. All patients with LABC who received NAC were collected in our institution from January 2013 to December 2017. Inclusion criteria for this study were: (1) An adult female patient over 18 years old; (2) Puncture biopsy confirmed unilateral invasive ductal carcinoma of the breast prior to NAC therapy; and (3) CE-MRI examination was performed within 2 weeks before NAC.

The exclusion criteria were as follows: (1) Patients who underwent the aspiration biopsy or accepted any endocrine or radiation therapy before MRI scans; (2) The baseline CE-MRI scan was performed more than 1 week before NAC; (3) Lesions were scarcely identified on MRI due to motion or other artifacts; (4) Neoadjuvant chemotherapy was not completed due to extraneous reasons; (5) Patients who did not perform surgical resection after the completion of NAC.
Figure 1 shows the identification, eligibility, and inclusion of patients in the study. A total of 55 patients were selected from initial identification of 83 patients after exclusion of patients who underwent other therapies before MRI $(n=13)$, those where more than 1 week had passed between MRI and NAC $(n=2)$, those with severe image artifacts $(n=5)$, incomplete NAC $(n=6)$, and those with no surgical resection after NAC $(n=2)$.

\section{Clinical and pathological data}

The patient's clinical data including patient's age, tumor size, tumor histopathologic type, molecular subtypes, lymph node invasion before NAC, tumor types (mass vs non-mass) in CE-MRI, and the regimen of NAC were retrieved from the hospital's medical record system. The pathological assessment of NAC was acquired from the pathology reports after breast-conserving surgery or mastectomy, which was completed by a pathologist with more than 10 years of working experience. The criteria of $\mathrm{pCR}$ were defined as the absence of residual invasive tumor in the surgical specimen (residual ductal carcinoma in situ could be present) and the absence of lymph node invasion in the ipsilateral sentinel node or lymph nodes removed during the axillary dissection.

\section{Image acquisition}

Each patient underwent DCE-MRI examination on a 3.0 Telsa MR scanner (Siemens, Erlangen, Germany) in the prone position with the use of a dedicated 16-channel bilateral phased-array breast coil for signal reception. Data was obtained for routine clinical practice. The DCE-MRI imaging protocol was as follows: $\mathrm{TR} / \mathrm{TE}=$ $4.51 \mathrm{~ms} / 1.61 \mathrm{~ms}$, section thickness, $1 \mathrm{~mm}$; flip angle, $10^{\circ}$. The matrix was $448 \times 448$; and NEX=6). One noncontrast and five contrast dynamic series were included into the DCE-MRI imaging. The fifth phase of imaging was selected into segmentation at $245 \mathrm{~s}$ after contrast injection. The gadolinium chelate was injected via the basilic vein with the dosage of $0.1 \mathrm{mmol} / \mathrm{kg}$ body weight, followed by a $10 \mathrm{~mL}$ flush of isotonic saline.

\section{Tumor segmentation}

Each tumor was segmented on enhanced T1-weighted images using a semi-automated segmentation tool in an open volumetric image analysis platform 3DQI (an open software platform for volumetric image analysis developed by the 3D quantitative imaging laboratory at Massachusetts General Hospital and Harvard Medical School (https://3dqi.mgh.harvard.edu), focusing on the prediction and assessment of the treatment response in clinical oncology). Each tumor was first identified and segmented on the axial plane by a breast radiologist with 3 years' experience and then verified by another breast 


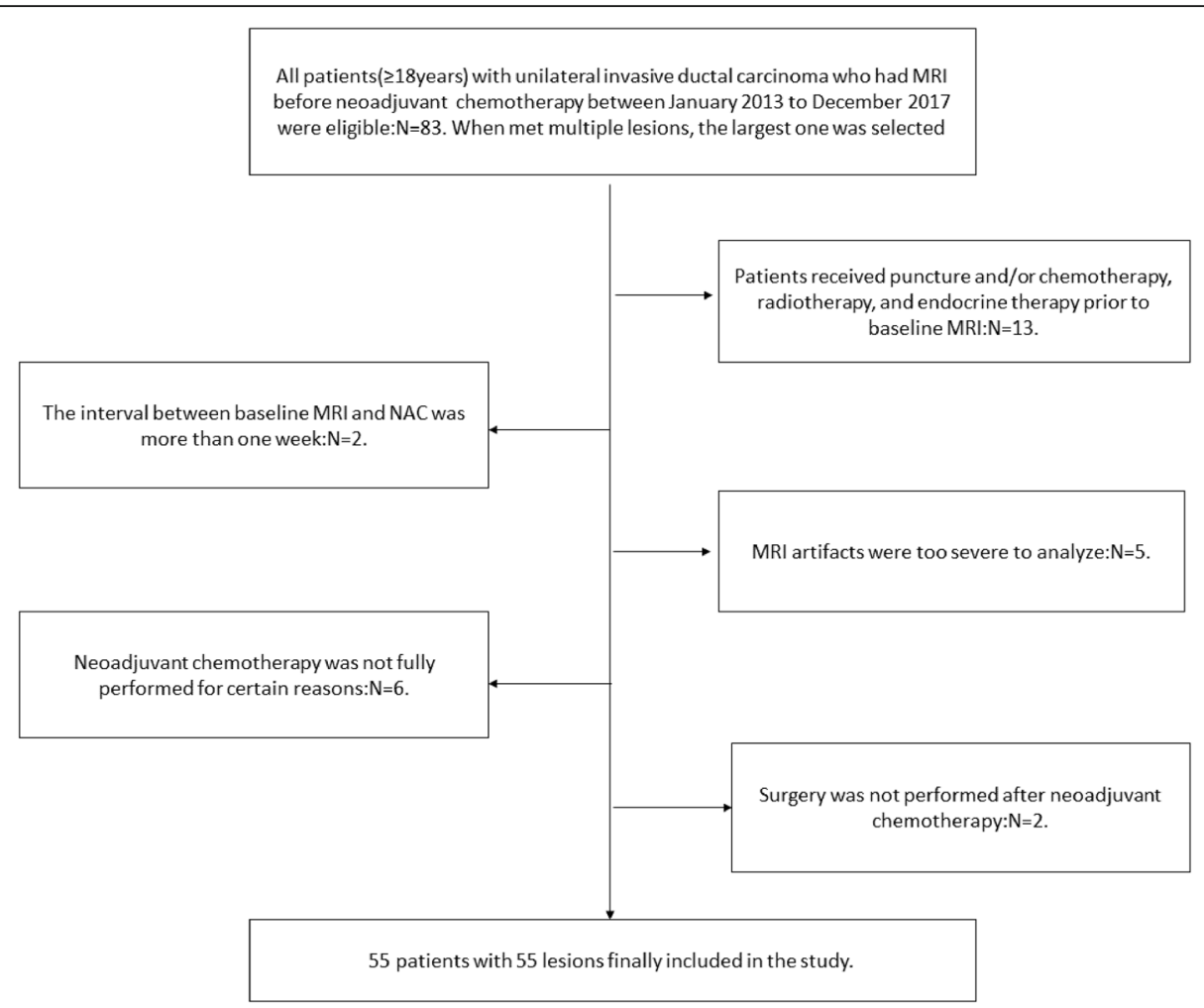

Fig. 1 Flow diagram of the patient selection in the study

radiologist with 7 years' experience. They were blinded to the pathological assessment of NAC after surgery. The corresponding sagittal and coronal planes of the tumor were referenced when the lesion was ambiguous in the axial plane. The volumes of interest (VOIs) of each tumor was determined by the consensus of both radiologists. In the case of multiple lesions in a patient, the largest detected lesion was selected.

\section{Radiomic analysis}

3DQI software (3D Quantitative Imaging Lab, Harvard Medical School) was utilized to texture calculation and radiomic analysis. Three groups of radiomic textures were calculated for the segmented lesions, including volumetric, peripheral textures, and wavelet-transformed textures. Volumetric textures were calculated in the entire volume of segmented lesion containing 5 categories: 11 shape features, 25 histogram statistical textures, 22 gray level co-occurrence matrix (GLCM) textures, 16 gray level run-length matrix (GLRLM) textures, and 14 gray level zone size matrix (GLZSM) textures. Peripheral textures were calculated in a $10 \mathrm{~mm}$ wide band region centered on the boundary of the segmented lesions, which covered the $5 \mathrm{~mm}$ inner region and $5 \mathrm{~mm}$ outer region separated by the lesion boundary. We calculated 77 volumetric textures except 11 shape features in the periphery region.
A 3D discrete and single-stage wavelet transform was used to decompose volumetric images into eight decomposed volumes of images, labeled as LLL, LLH, LHL, LHH, HLL, HLH, HHL and HHH, where $\mathrm{L}$ and $\mathrm{H}$ are low- and high-frequency signals, respectively. For example, LLH is a volume of images transformed by using the low-pass filters on the $\mathrm{X}$ and $\mathrm{Y}$ axis, and a $\mathrm{Z}$-axis high-pass filter. In the eight decomposed volumes of images, 3DQI calculated five categories of volumetric textures with the exception of the shape features in the segmented lesion VOIs, which resulted in a total of 616 (8X77) wavelet-transformed texture features for each VOI.

A random forest (RF) was applied [17] to predict the response of $\mathrm{pCR}$ to NAC using tumor texture features calculated from the pre-operative CE-MRI. RF is a machine learning classifier, which can prevent over-fitting of the data (due to a large number of radiomic features) by injecting randomness into the training of the trees and combining the output of multiple random trees into the final classifier. Thus, a random forest is known to perform consistently well in high-dimensional data compared with other classification algorithms [17]. We trained six RF models to the prediction of pCR by using six combinations of three groups of radiomic textures along with the clinical outcomes. Each RF classification model had 100 trees with a node size of 1 . The number 
of features for each tree is the square root of the total number of features in each model (rounded up). To avoid over-fitting, RF randomly chooses a subset of features (feature bagging) with respect to the number of features to grow each tree, and randomly sampled the subset of the bootstrapped data (sample bagging). The six radiomics combinations were Model I: volumetric textures, Model II: volumetric+peripheral textures, Model III: wavelet textures, Model IV: volumetric+wavelet textures, Model V: peripheral+wavelet textures, and Model VI: volumetric+peripheral+wavelet textures.

For the selection of important textures in each model, we adopted a two-round feature selection scheme to select the optimal features for each model. First, the importance scores calculated by the Boruta algorithm were used for a rapid reduction of texture dimensionality [18]. The Boruta algorithm is a feature ranking and selection algorithm based on the random forests algorithm, which identifies all features which are either strongly or weakly relevant to the decision variable. The importance of a feature is defined by the loss of classification accuracy caused by the random permutation of feature values between objects. Non-relevant features were rejected by using $\mathrm{Z}$ score cutoff of less than 0.01. An initial RF model was established after the first round by including all relevant features. At the second round, an iterative culling-out algorithm was used to refine the model [19]. In each iteration, we calculated the prediction performance of the RF model by removing one of the textures, i.e. the AUC value of the ROC curve. If the AUC value using one-less texture parameter is higher than that of current RF model, the model corresponding to the maximum AUC value was selected. This iteration was completed until no AUC values were higher than that of the current model.

To reduce the bias that may be caused by an unbalanced number of positive and negative samples, we applied the SMOTE (Synthetic Minority Oversampling Technique) resampling method $[20,21]$, which combines informed oversampling of the minority class (patients with small number of tumors) with random undersampling of the majority class (patients with large number of tumors) to balance the samples between different patient groups. All radiomic features in each patient group were

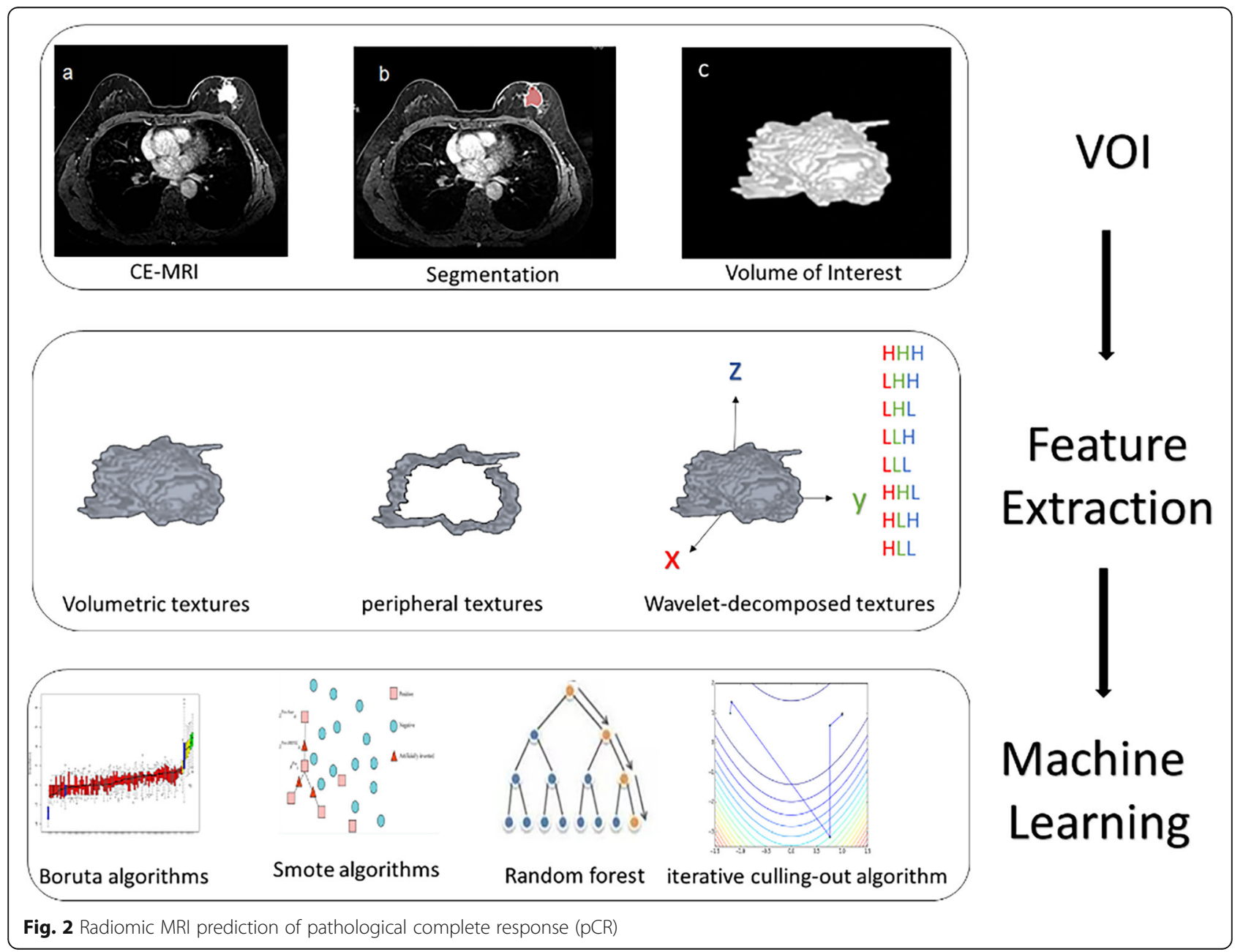


resampled to 50 tumor radiomic samples by SMOTE method, which resulted in 100 samples including 50 pCR and 50 nonpCR samples. A 10-fold cross-validation method was applied to train and validate the model through $n=100$ repetitions. The model performance was compared by using the AUC values, represented by mean \pm SD. Figure 2 shows the pipeline of our RF models for prediction of $\mathrm{pCR}$.

\section{Statistical analysis}

All statistical analyses were performed in SPSS version 19.0. A Chi square test or Fisher's exact test was used for the nominal variable. A Mann-Whitney $U$ test was used for the unordered categorical variable. A student's $t$ test was used for the continuous variable. A $p$-value less than 0.05 was considered statistically significant.

\section{Results}

\section{Clinical and pathological data}

Fifty-five female patients aged 25 to 75 years (mean age = $50.4 \pm 12.2$ years) were enrolled in the study which included 49 patients with a single breast tumor and 6 patients with multiple tumors. All patients were diagnosed with invasive ductal carcinoma by pre-NAC puncture biopsy and received NAC prior to surgical resection. Clinical and pathological data of the study were listed in Table 1 . The pCR rate was $30.9 \%(17 / 55)$ (mean age $=$ $50.7 \pm 9.4$ years), whereas non-pCR rate was $69.1 \%$ (38/ 55) (mean age $=49.5 \pm 10.4$ years). The median maximum diameters of the lesions were $2.6 \mathrm{~cm}$ (range: $2.3-$ $3.7 \mathrm{~cm}$ ) and $4.2 \mathrm{~cm}$ (range $3.1-5.4 \mathrm{~cm}$ ) in the pCR and the non-pCR group, respectively; and the mean diameters were $2.9 \pm 1.1 \mathrm{~cm}$ and $4.3 \pm 1.9 \mathrm{~cm}$, respectively. Except for the maximum diameter $(p=0.002)$, there were no statistically significant differences between $\mathrm{pCR}$ and non-pCR groups of patients. Figure 3 demonstrates the segmentation of breast lesions on CE-MRI.

\section{Radiomic models}

After applying our feature selection method to three groups of MRI radiomic textures, we identified 5 volumetric texture features, 3 peripheral texture features, and 3 wavelet texture features, respectively, for the prediction of pCR. Table 2 lists the six models by their combinations of the three groups of selected features. The AUCs of the six models for the prediction of pCR were $0.816 \pm 0.033$ (Model I: volumetric textures), $0.823 \pm 0.020$ (Model II: volumetric + peripheral textures), $0.888 \pm 0.025$ (Model III: wavelet textures), $0.876 \pm 0.015$ (Model IV: volumetric + wavelet textures), $0.885 \pm 0.030$ (Model V: peripheral + wavelet textures), and $0.874 \pm 0.019$ (Model VI: volumetric + peripheral + wavelet textures). Figure 4 shows the ROCs and AUC values of the six models.
Table 1 Clinical and pathological data in the study

\begin{tabular}{llll}
\hline & $\mathrm{pCR}$ & Non-pCR & P-value \\
\hline No. of patients & 17 & 38 & $\mathrm{~N} / \mathrm{A}$ \\
Age(y) & & & \\
$\quad$ Median(range) & $50(37-70)$ & $48(25-68)$ & $\mathrm{N} / \mathrm{A}$ \\
$\quad$ Mean \pm SD & $50.7 \pm 9.4$ & $49.5 \pm 10.4$ & 0.676 \\
Enhancement Type, No. (\%) & & & 0.506 \\
$\quad$ Masslike & $11(64.7)$ & $23(60.5)$ & \\
Non-masslike & $6(35.3)$ & $15(39.5)$ & \\
Max-D(cm)* & & & \\
Median(range) & $2.6(2.3-3.7)$ & $4.2(3.1-5.4)$ & N/A \\
Mean \pm SD & $2.9 \pm 1.1$ & $4.3 \pm 1.9$ & 0.002 \\
Subtype, No. (\%) & & & 0.493 \\
Luminal A & $5(29.4)$ & $17(44.7)$ & \\
Luminal B & $2(11.8)$ & $7(18.4)$ & \\
Her-2 & $5(29.4)$ & $8(21.1)$ & \\
TNBC & $5(29.4)$ & $6(15.8)$ & \\
Regimen, No. (\%) & & & \\
EC+ Taxol & $4(23.5)$ & $14(36.8)$ & \\
FEC+ Taxol & $7(41.2)$ & $15(39.5)$ & \\
AC+ Taxol & $2(11.8)$ & $6(15.8)$ & \\
Others & $4(23.5)$ & $3(7.9)$ & \\
\hline
\end{tabular}

NOTE. P-values were calculated by T-test or Mann-Whitney U test for Age, Max-D, from Chi-square test or Fisher's exact test for Enhancement type, Subtype, Regimen

Abbreviations: Max-D Maximum- diameter, Her-2 Human epidermal growth factor receptor 2, TNBC Triple negative breast cancer, $E$ epirubicin; $C$ cyclophosphamide, Taxol paclitaxel; $F$ 5-fluoroucil. A doxorubicin. N/A Not available

The performance (AUC, accuracy, sensitivity, and specificity) of four models with wavelet textures (Models III, IV, V, and IV) were statistically significantly better than those without wavelet textures (Model I and II). The models by inclusion of peripheral textures did not show significant improvements in performance compared to those exclusion of peripheral textures (Model I vs Model II, $p=0.985$; Model III vs Model V, $p=1.000$ ). Also, the addition of either volumetric textures or peripheral textures or both to the wavelet textures (Models IV vs Model III, $p=0.891$; Model V vs Model III, $p=$ 1.000; Model VI vs Model III, $p=0.809$ ) did not yield any improvements in performance compared to the model with wavelet textures only (Model III). Figure 5 plots the AUCs of the six models, and Table 3 lists the $p$-values among the six models.

\section{Discussions}

Several studies have investigated radiomics models in the prediction of the response of breast cancer to NAC on CE-MRI [10-12, 22], as shown in Additional file 1: Table S1. However, the performance of these models varied due 

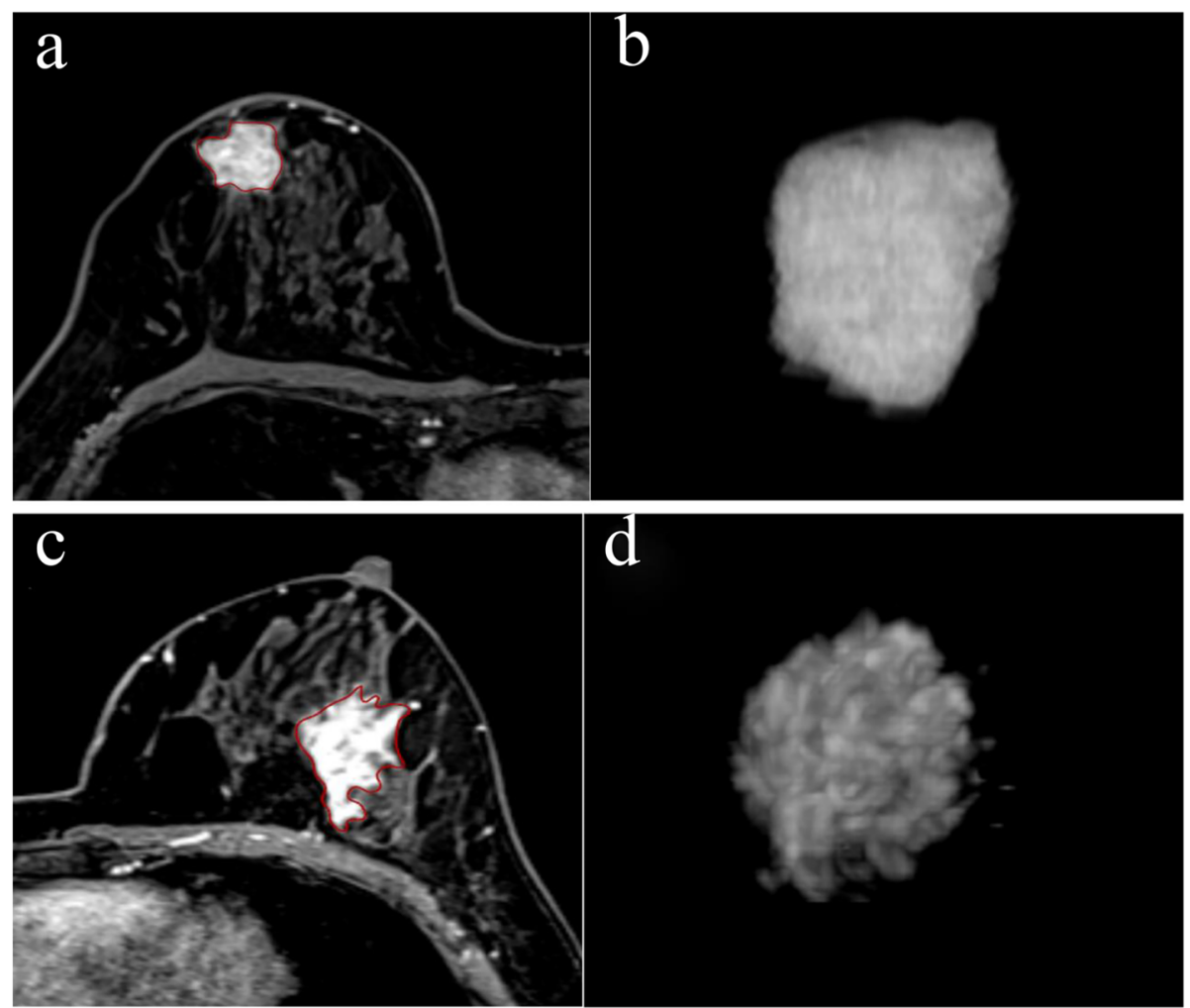

Fig. 3 Segmentation of breast lesions on CE-MRI. Images $\mathbf{a}-\mathbf{b}$ show the right invasive breast cancer that was non-pCR after NAC. Images $c-d$ show the left invasive breast cancer that was PCR after NAC. a, c Segmentation of breast lesions on CE-MRI. b, d 3D imaging of VOIs

Table 2 Textures and performance (AUC, Accuracy, Sensitivity and Specificity) of six RF models

\begin{tabular}{|c|c|c|c|c|c|c|}
\hline & \multicolumn{6}{|l|}{ RF Models } \\
\hline & I & $\|$ & III & IV & $\mathrm{V}$ & $\mathrm{VI}$ \\
\hline Features & Volumetric & $\begin{array}{l}\text { Volumetric + } \\
\text { Peripheral }\end{array}$ & Wavelet & $\begin{array}{l}\text { Volumetric }+ \\
\text { Wavelet }\end{array}$ & $\begin{array}{l}\text { Peripheral + } \\
\text { Wavelet }\end{array}$ & $\begin{array}{l}\text { Volumetric + Peripheral + } \\
\text { Wavelet }\end{array}$ \\
\hline Selected Features & $\begin{array}{l}\text { GLZSM_salgle } \\
\text { GLCM_homo1 } \\
\text { GLCM_diffEntro } \\
\text { GLCM__ } \\
\text { dissimilar } \\
\text { SHAPE_- } \\
\text { surfaceArea }\end{array}$ & $\begin{array}{l}\text { GLZSM_salgle } \\
\text { GLCM_homo1 } \\
\text { GLCM_diffEntro } \\
\text { GLCM_dissimilar } \\
\text { Bndry_RL_rln } \\
\text { Bndry_GLZSM_ } \\
\text { salgle } \\
\text { Bndry_GLCM__ } \\
\text { contrast } \\
\text { SHAPE_- } \\
\text { surfaceArea }\end{array}$ & $\begin{array}{l}\text { LHH_GLZSM_zp } \\
\text { LLH_GLCM_ } \\
\text { infoCorr1 } \\
\text { HHH_GLCM_ } \\
\text { correlation }\end{array}$ & $\begin{array}{l}\text { GLZSM_salgle } \\
\text { GLCM_homo1 } \\
\text { GLCM_diffEntro } \\
\text { GLCM_dissimilar } \\
\text { HHH_GLCM_ } \\
\text { correlation } \\
\text { LHH_GLZSM_zp } \\
\text { LLH_GLCM_ } \\
\text { infoCorr1 } \\
\text { SHAPE_surfaceArea }\end{array}$ & $\begin{array}{l}\text { Bndry_RL_rln } \\
\text { Bndry_GLZSM_ } \\
\text { salgle } \\
\text { Bndry_GLCM_ } \\
\text { contrast } \\
\text { HHH_GLCM_ } \\
\text { correlation } \\
\text { LHH_GLZSM_zp } \\
\text { LLH_GLCM_ } \\
\text { infoCorr1 }\end{array}$ & $\begin{array}{l}\text { SHAPE_surfaceArea } \\
\text { GLZSM_salgle } \\
\text { GLCM_homo1 } \\
\text { GLCM_diffEntro } \\
\text { GLCM_dissimilar } \\
\text { Bndry_RL_rln } \\
\text { Bndry_GLZSM_salgle } \\
\text { Bndry_GLCM_contrast } \\
\text { HHH_GLCM_correlation } \\
\text { LHH_GLZSM_zp } \\
\text { LLH_GLCM_infoCorr1 }\end{array}$ \\
\hline $\mathrm{AUC}($ mean $\pm \mathrm{SD})$ & $0.816 \pm 0.033$ & $0.823 \pm 0.020$ & $0.888 \pm 0.025$ & $0.876 \pm 0.015$ & $0.885 \pm 0.030$ & $0.874 \pm 0.019$ \\
\hline $\begin{array}{l}\text { Accuracy (mean } \pm \\
\text { SD) }\end{array}$ & $0.747 \pm 0.022$ & $0.751 \pm 0.0150$ & $0.810 \pm 0.030$ & $0.781 \pm 0.028$ & $0.797 \pm 0.032$ & $0.787 \pm 0.024$ \\
\hline $\begin{array}{l}\text { Sensitivity } \\
\text { (mean } \pm \text { SD) }\end{array}$ & $0.676 \pm 0.043$ & $0.684 \pm 0.043$ & $0.762 \pm 0.035$ & $0.730 \pm 0.049$ & $0.770 \pm 0.034$ & $0.727 \pm 0.045$ \\
\hline $\begin{array}{l}\text { Specificity } \\
\text { (mean } \pm S D)\end{array}$ & $0.812 \pm 0.023$ & $0.798 \pm 0.021$ & $0.845 \pm 0.031$ & $0.818 \pm 0.037$ & $0.818 \pm 0.047$ & $0.830 \pm 0.035$ \\
\hline
\end{tabular}



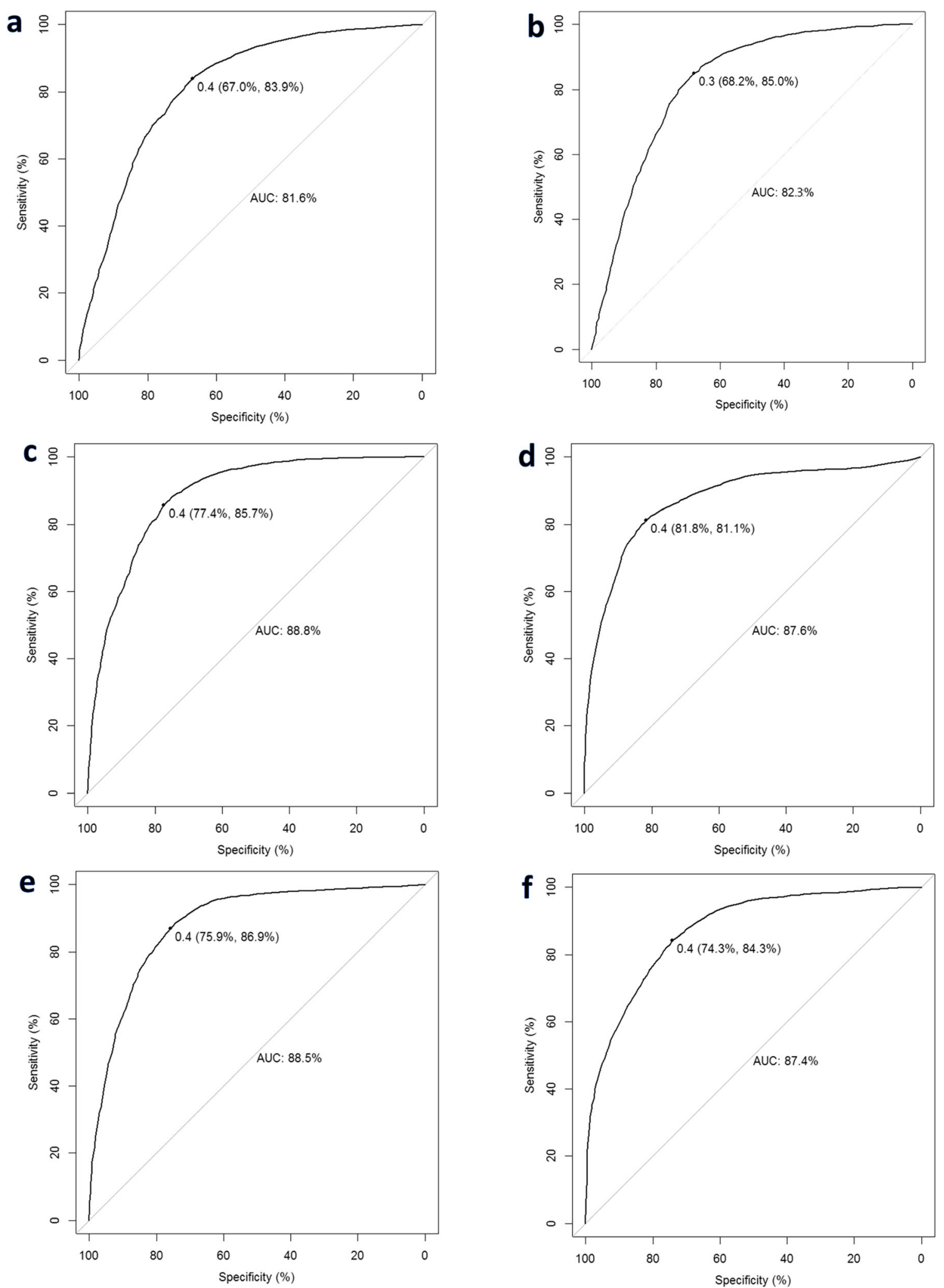

Fig. 4 Receiver operating characteristic (ROC) curves of the six RF models: a Model I: volumetric textures, b Model II: volumetric + peripheral textures, c Model III: wavelet textures, d Model IV: volumetric + wavelet textures, e Model V: peripheral + wavelet textures, and $\mathbf{f}$ Model VI: volumetric + peripheral + wavelet textures 


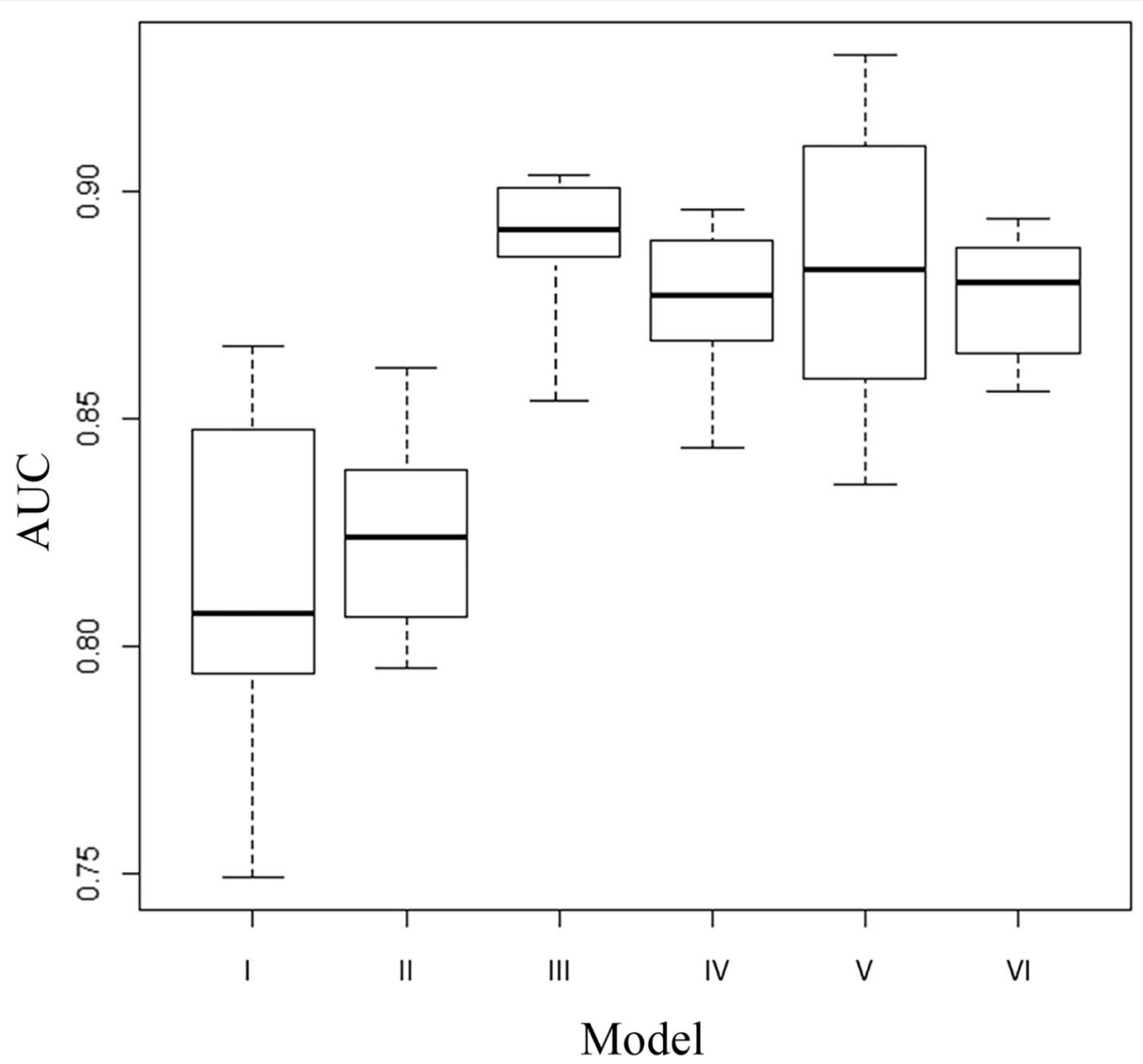

Fig. 5 Boxplots of the prediction performance (AUC, area under ROC curve) of six radiomics models

to the different types of radiomic features extracted and different types of VOIs applied, intratumoral or peritumoral regions. This study investigated six radiomics models composed of three groups of textures, volumetric textures, peripheral textures, and wavelet-transformed textures, for prediction of pCR to NAC in breast CE-MRI.

Among 88 volumetric textures, five features were selected as important features, of which three textures were chosen from GLCM features. Prior studies have demonstrated that GLCM may be associated with intratumoral heterogeneity, and high intratumoral heterogeneity may be associated with poor prognosis [23, 24]. GLCM features were also applied to predict

Table 3 Comparison of p-values of AUCs between 6 models

\begin{tabular}{|c|c|c|c|c|c|c|}
\hline Models & 1 & $\|$ & III & IV & V & $\mathrm{Vl}$ \\
\hline 1 & N/A & 0.985 & $<0.001$ & $<0.001$ & $<0.001$ & $<0.001$ \\
\hline$\|$ & & N/A & $<0.001$ & $<0.001$ & $<0.001$ & 0.001 \\
\hline III & & & N/A & 0.891 & 1.000 & 0.809 \\
\hline IV & & & & N/A & 0.968 & 1.000 \\
\hline V & & & & & N/A & 0.924 \\
\hline VI & & & & & & N/A \\
\hline
\end{tabular}

NOTE: P-values are calculated from the T-test chemotherapy response to triple negative breast cancer [22], which was consistent with the results of our study. In addition, tumor shape features may improve the prediction of prognosis of LABC underwent NAC [25]. For instance, tumor size and tumor surface characteristics were related to the effectiveness of NAC [26, 27]. In the group of peripheral texture features, three features were selected, one from each of the GLCM, GLZSM, and RL families, respectively, representing the homogeneity and heterogeneity of peripheral tumor regions $[9,28]$. The wavelet transformation decomposes images into high frequency (heterogeneity) and low frequency (homogeneity) for both intratumoral and peritumoral regions [29]. The HHH_GLCM family highlighted the features of the tumor boundary and any internal inhomogeneity. The LLH_GLCM family revealed the intra-slice homogeneity and inter-slice inhomogeneity characterization. Peritumoral textures may be characterized by the high frequency signals in the tumor boundary regions in the wavelet-transformed images [11], whereas, intratumoral textures may be characterized by the low frequency domains in wavelet-decomposed images.

In our study, four models with wavelet-transformed textures (Model III to VI) outperformed Model I and II 
without wavelet textures in the prediction of $\mathrm{pCR}$ to NAC of LABC $(p<0.001)$. This indicated that the inclusion of wavelet-transformed features may improve the performance of the prediction models, which is consistent with the results of Imon Banerjee et al. [30] In general, lesion edges are related to the high-frequency signals in the wavelet-transformed images. Prior studies have demonstrated that the addition of peritumoral texture features optimized the performance for predicting $\mathrm{pCR}$ of NAC [11]. In our study, Model II achieved a better performance than Model I after the addition of peritumoral texture features, but without statistical significance $(p=0.892)$. On the other hand, in the comparison of Models III to VI, we observed that the inclusion of the peritumoral texture features into the wavelet-decomposed textures (Model VI) did not show significant improvements in the model (Model III). This revealed that characterization from intertumoral and peritumoral textures may be contained in the wavelet-decomposed textures. The wavelet-transformed textures achieved the best performance for radiomic MRI prediction of the pCR of NAC for breast cancer. Thus, wavelet-transformed textures may be sufficient to predict pCR of NAC without calculation of textures separately in the intertumoral and peritumoral regions.

A 3D discrete wavelet transformation decomposes images into one approximation and seven detailed images, which are mutually orthogonal sets of wavelets, representing the low-frequency (smooth such as homogeneous intertumoral region) and high-frequency (non-smooth such as tumor boundaries or heterogeneous intertumoral region) contents of the images, respectively, which are not affected by motion or orientation. On the other hand, Gabor wavelets are claimed to be sensitive for detecting the local texture features corresponding to specific orientations, allowing optimally extracted information such as retinal blood vessels and vessel diameter [31, 32]. Nathaniel M. Braman et al. applied Gabor wavelet in both intratumoral and peritumoral regions to extract detailed edge information [11]. The Gabor wavelet features based on manually selected regions tend to show an unreliable performance as manual selection leads to loss of tumor shape information due to interobserver variability.

Despite the findings presented herein, this study had three major limitations. Firstly, the retrospective nature of the study lacks external validation outside a single institution. Secondly, sampling bias may exist as a result of the small sample size in our study. The small sample size was caused by the strict inclusion and exclusion criteria. Therefore, the results in the present investigation also need to be verified by further studies. The third limitation is the unbalanced sample sizes of the experimental group and the control group. Although a SMOTE algorithm was used to balance the data, some bias may still exist between the two groups.

\section{Conclusions}

Our study demonstrated that wavelet-transformed textures outperformed intratumoral and peritumoral textures for radiomic MRI prediction of $\mathrm{pCR}$ to NAC for patients with LABC. Therefore, the method presented in this study may provide a potential surrogate for the accurate prediction of the clinical outcomes of NAC, resulting more effective treatment.

\section{Supplementary information}

Supplementary information accompanies this paper at https://doi.org/10. 1186/s12885-020-6523-2.

Additional file 1 Table S1. Comparison between our results and the

recent obtained results.

\section{Abbreviations}

AUC: Area under curve; CE-MRI: Contrast-enhanced magnetic resonance imaging; NAC: Neoadjuvant chemotherapy; pCR: Pathological complete response; ROC: Receiver operating characteristic; VOI: Volume of interest

\section{Acknowledgements}

The authors thank all the medical staff who contributed to the maintenance of the medical record database.

\section{Authors' contributions}

All authors contributed to this paper. MX and WC designed the study. JZhou and $J$ wrote the manuscript. JZhou and CG collected patients that meet our inclusion criteria. $X L$ downloaded the images of these patients and evaluated the quality of data. JZeng and CZ segmented lesions. Image analysis and statistical analyses were done by JL and WC. MX, WC, JZhou and CG revised the manuscript. MX and WC approved the final version. All authors have read and approved the manuscript.

\section{Funding}

This study was funded by National Natural Science Foundation of China (grant number 61602419), the Chinese medicine research foundation project of Zhejiang Province (grant number 2018ZA037) of China, Zhejiang provincial medicine and health discipline platform project (grant number 2018RC058), Zhejiang provincial health department platform backbone project (grant number 2016147237). The funders had no role in the design of the study and collection, analysis, and interpretation of data and in writing the manuscript.

\section{Availability of data and materials}

The data that support the findings of this study are available from the corresponding author upon reasonable requests.

\section{Ethics approval and consent to participate}

All procedures performed in studies involving human participants were in accordance with the ethical standards of the institutional and/or national research committee and with the 1964 Helsinki declaration and its later amendments or comparable ethical standards.

The Ethics Committee of the First Affiliated Hospital of Zhejiang Chinese Medical University has approved this retrospective study, in which informed consent was waived, but patient confidentiality was protected.

\section{Consent for publication}

Not applicable.

\section{Competing interests}

Wenli Cai: Shareholder of IQ Medical Imaging LLC.

The other authors have no relevant conflicts of interest to disclose.

\section{Author details}

${ }^{1}$ Department of Radiology, The First Affiliated Hospital of Zhejiang Chinese Medical University, 54 Youdian Road, Shangcheng District, Hangzhou 310006, 
People's Republic of China. ${ }^{2}$ The First Clinical Medical College of Zhejiang Chinese Medical University, Hangzhou, China. ${ }^{3}$ Ningbo First Hospital, Ningbo, China. ${ }^{4}$ Department of Radiology, Massachusetts General Hospital and Harvard Medical School, 25 New Chardon St., 400C, Boston, MA 02114, USA. ${ }^{5}$ The Third Affiliated Hospital of Zhejiang Chinese Medical University, Hangzhou, China.

Received: 5 September 2019 Accepted: 7 January 2020

Published online: 05 February 2020

\section{References}

1. Harbeck N, Gnant M. Breast cancer. Lancet (London, England). 2017; 389(10074):1134-50. https://doi.org/10.1016/S0140-6736(16)31891-8.

2. Siegel RL, Miller KD, Jemal A. Cancer statistics, 2016. CA Cancer J Clin. 2016; 66(1):7-30. https://doi.org/10.3322/caac.21332.

3. Rivera-Rodriguez N, Cabanillas F, Lawrenson L, et al. Abstract P3-14-17: results of a novel neoadjuvant chemotherapy (NAC) for breast cancer. Cancer Res. 2013;73(24 Supplement):P3-14-17-P3-14-17). https://doi.org/10. 1158/0008-5472.SABCS13-P3-14-17.

4. Santamaria G, Bargallo X, Fernandez PL, Farrus B, Caparros X, Velasco M. Neoadjuvant systemic therapy in breast Cancer: Association of Contrastenhanced MR imaging findings, diffusion-weighted imaging findings, and tumor subtype with tumor response. Radiology. 2017;283(3):663-72. https:// doi.org/10.1148/radiol.2016160176.

5. Schott AF, Hayes DF. Defining the benefits of neoadjuvant chemotherapy for breast cancer. J Clin Oncol. 2012;30(15):1747-9. https://doi.org/10.1200/ JCO.2011.41.3161.

6. von Minckwitz G, Untch M, Blohmer J-U, et al. Definition and impact of pathologic complete response on prognosis after neoadjuvant chemotherapy in various intrinsic breast cancer subtypes. J Clin Oncol. 2012; 30(15):1796-804. https://doi.org/10.1200/JCO.2011.38.8595.

7. Earl H, Provenzano E, Abraham J, et al. Neoadjuvant trials in early breast cancer: pathological response at surgery and correlation to longer term outcomes - what does it all mean? BMC Med. 2015;13:234. https://doi.org/ 10.1186/s12916-015-0472-7.

8. Sparano JA, Gray RJ, Makower DF, et al. Prospective validation of a 21-gene expression assay in breast Cancer. N Engl J Med. 2015;373(21):2005-14. https://doi.org/10.1056/NEJMoa1510764.

9. Thibault G, Tudorica A, Afzal A, et al. DCE-MRI Texture Features for Early Prediction of Breast Cancer Therapy Response. Tomography. 2017;3(1):2332. https://doi.org/10.18383/j.tom.2016.00241.

10. Chamming's $F$, Ueno $Y$, Ferre $R$, et al. Features from computerized texture analysis of breast cancers at pretreatment MR imaging are associated with response to Neoadjuvant chemotherapy. Radiology. 2018;286(2):412-20. https://doi.org/10.1148/radiol.2017170143.

11. Braman NM, Etesami M, Prasanna P, et al. Intratumoral and peritumoral radiomics for the pretreatment prediction of pathological complete response to neoadjuvant chemotherapy based on breast DCE-MRI. Breast Cancer Res. 2017;19(1):57. https://doi.org/10.1186/s13058-017-0846-1.

12. Fan M, Wu G, Cheng H, Zhang J, Shao G, Li L. Radiomic analysis of DCE-MRI for prediction of response to neoadjuvant chemotherapy in breast cancer patients. Eur J Radiol. 2017;94:140-7. https://doi.org/10.1016/j.ejrad.2017.06. 019.

13. Wu J, Gong G, Cui Y, Li R. Intratumor partitioning and texture analysis of dynamic contrast-enhanced (DCE)-MRI identifies relevant tumor subregions to predict pathological response of breast cancer to neoadjuvant chemotherapy. J Magn Reson Imaging. 2016;44(5):1107-15. https://doi.org/ 10.1002/jmri.25279.

14. Teruel JR, Heldahl MG, Goa PE, et al. Dynamic contrast-enhanced MRI texture analysis for pretreatment prediction of clinical and pathological response to neoadjuvant chemotherapy in patients with locally advanced breast cancer. NMR Biomed. 2014;27(8):887-96. https://doi.org/10.1002/nbm. 3132.

15. Majeed Alneamy JS, A Hameed Alnaish Z, Mohd Hashim SZ, Hamed Alnaish RA. Utilizing hybrid functional fuzzy wavelet neural networks with a teaching learning-based optimization algorithm for medical disease diagnosis. Comput Biol Med. 2019;112:103348. https://doi.org/10.1016/j. compbiomed.2019.103348.

16. Das DK, Dutta PK. Efficient automated detection of mitotic cells from breast histological images using deep convolution neutral network with wavelet decomposed patches. Comput Biol Med. 2019;104:29-42. https://doi.org/10. 1016/j.compbiomed.2018.11.001.

17. Svetnik V, Liaw A, Tong C, Culberson JC, Sheridan RP, Feuston BP. Random forest: a classification and regression tool for compound classification and QSAR modeling. J Chem Inf Comput Sci. 2003;43(6):1947-58. https://doi.org/ 10.1021/ci034160g.

18. Rahman QA, Janmohamed T, Clarke H, Ritvo P, Heffernan J, Katz J. Interpretability and class imbalance in prediction models for pain volatility in manage my pain app users: analysis using feature selection and majority voting methods. JMIR Med Inform. 2019;7(4):e15601. https://doi.org/10.2196/ 15601.

19. Udroiu I, Sgura A. Quantitative relationships between acentric fragments and micronuclei: new models and implications for curve fitting. Int J Radiat Biol. 2019:1-9. https://doi.org/10.1080/09553002.2020.1683638.

20. Nakamura M, Kajiwara Y, Otsuka A, Kimura H. LVQ-SMOTE - learning vector quantization based synthetic minority over-sampling technique for biomedical data. BioData Min. 2013;6(1):16. https://doi.org/10.1186/17560381-6-16.

21. Chawla N, Bowyer K, Hall L, Kegelmeyer W. SMOTE: synthetic minority oversampling technique. J Artif Intell Res. 2002;16:321-57. https://doi.org/10. 1613/jair.953.

22. Golden DI, Lipson JA, Telli ML, Ford JM, Rubin DL. Dynamic contrastenhanced MRI-based biomarkers of therapeutic response in triple-negative breast cancer. J Am Med Inform Assoc. 2013;20(6):1059-66. https://doi.org/ 10.1136/amiajnl-2012-001460.

23. Depeursinge A, Foncubierta-Rodriguez A, Van De Ville D, Muller H. Threedimensional solid texture analysis in biomedical imaging: review and opportunities. Med Image Anal. 2014;18(1):176-96. https://doi.org/10.1016/j. media.2013.10.005.

24. Kim J-H, Ko ES, Lim Y, et al. Breast Cancer heterogeneity: MR imaging texture analysis and survival outcomes. Radiology. 2017;282(3):665-75. https://doi.org/10.1148/radiol.2016160261.

25. Pickles MD, Lowry M, Gibbs P. Pretreatment prognostic value of dynamic contrast-enhanced magnetic resonance imaging vascular, texture, shape, and size parameters compared with traditional survival indicators obtained from locally advanced breast Cancer patients. Investig Radiol. 2016;51(3): 177-85. https://doi.org/10.1097/RLI.0000000000000222.

26. Flynn C, Gullo G, Walshe J, et al. Use of contrast-enhanced magnetic resonance imaging (MRI) to predict pathological response after trastuzumab (T) - based neoadjuvant chemotherapy (NAC) for HER2-positive breast cancer (HER2BrCa). Eur J Cancer. 2018;92:S83. https://doi.org/10.1016/S09598049(18)30477-5.

27. Partridge SC, Gibbs JE, Lu Y, et al. MRI measurements of breast tumor volume predict response to neoadjuvant chemotherapy and recurrence-free survival. AJR Am J Roentgenol. 2005;184(6):1774-81. https://doi.org/10.2214/ ajr.184.6.01841774.

28. Wu J, Li B, Sun X, et al. Heterogeneous enhancement patterns of tumoradjacent parenchyma at MR imaging are associated with Dysregulated signaling pathways and poor survival in breast Cancer. Radiology. 2017; 285(2):401-13. https://doi.org/10.1148/radiol.2017162823.

29. Wang $Y$, Che $X$, Ma S. Nonlinear filtering based on 3D wavelet transform for MRI denoising. EURASIP J Adv Signal Process. 2012;2012(1):40. https://doi. org/10.1186/1687-6180-2012-40.

30. Banerjee I, Malladi S, Lee D, et al. Assessing treatment response in triplenegative breast cancer from quantitative image analysis in perfusion magnetic resonance imaging. J Med imaging (Bellingham, Wash). 2018;5(1): 11008. https://doi.org/10.1117/1.JMl.5.1.011008.

31. Rangayyan RM, Banik S, Chakraborty J, Mukhopadhyay S, Desautels JEL. Measures of divergence of oriented patterns for the detection of architectural distortion in prior mammograms. Int J Comput Assist Radiol Surg. 2013;8(4):527-45. https://doi.org/10.1007/s11548-012-0793-3.

32. Yoshikawa R, Teramoto A, Matsubara T, Fujita H. In: Fujita H, Hara T, Muramatsu $C$, editors. Automated detection of architectural distortion using improved adaptive Gabor filter BT - breast imaging. Cham: Springer International Publishing; 2014. p. 606-11.

\section{Publisher's Note}

Springer Nature remains neutral with regard to jurisdictional claims in published maps and institutional affiliations. 